\title{
Exact Solutions for (2+1)-dimensional Nonlinear Schrödinger Equation Based on Modified Extended tanh Method
}

\author{
Wang Juan ${ }^{1, *}$, Wang Yudi ${ }^{2}$ and Xu Qian ${ }^{3}$ \\ ${ }^{1}$ College of Liberal Education, Hefei Information Technology University, Hefei, Anhui, 231200, \\ P.R. China; e-mail:wj92617@126.com \\ ${ }^{2}$ College of Liberal Education, Hefei Information Technology University, Hefei, Anhui, 231200, \\ P.R. China; e-mail: 303872016@qq.com \\ ${ }^{3}$ College of Liberal Education, Hefei Information Technology University, Hefei, Anhui, 231200, \\ P.R. China; e-mail: xuq0566@163.com \\ * Corresponding author
}

\begin{abstract}
In this paper, modified extended tanh method is used to construct more general exact solutions of a (2+1)-dimensional nonlinear Schrödinger equation. With the aid of Maple and Matlab software, we obtain exact explicit kink wave solutions, peakon wave solutions, periodic wave solutions and so on and their images.
\end{abstract}

\section{Introduction}

It is well known that Schrödinger equation is one of the most basic equations of quantum mechanics. It reflects the state of micro particle changing with time. As it is a powerful tool for solving non relativistic problems in atomic physics, it was widely used in the fields of atomic, molecular, solid state physics, nuclear physics, chemistry and so on. Recently, searching and constructing exact solutions of nonlinear partial differential

Received: March 9, 2019; Accepted: April 12, 2019

2010 Mathematics Subject Classification: 34C60, 35C07, 35Q55.

Keywords and phrases: Schrödinger equation, modified extended tanh method, kink wave solution, peakon wave solution, periodic wave solution.

Copyright () 2019 Wang Juan, Wang Yudi and Xu Qian. This is an open access article distributed under the Creative Commons Attribution License, which permits unrestricted use, distribution, and reproduction in any medium, provided the original work is properly cited. 
(NLPD) equation is very meaningful for it can describe the problems of mechanics, control process, ecological and economic system, chemical recycling system and epidemiological. In the past several decades, much efforts have been on this aspect and many useful methods have been proposed such as inverse scattering method, Jacobi elliptic function method, F-expansion method, Darboux transform, the sine-cosine method and the tanh method. The tanh method is widely used as it can find exact as well as approximate solutions in a systematic way. Subsequently, Fan has proposed an extended tanh method and obtained the travelling wave solutions that cannot be obtained by the tanh method. Based on this approach, we employed the modified extended tanh method to construct a series of exact travelling wave solutions of a (2+1)-dimensional nonlinear Schrödinger (NLS) equation as

$$
i u_{t}+\alpha u_{x x}+\beta u_{y y}+r\left|u^{2}\right| u=0 .
$$

\section{The tanh Method}

Here we review the modified extended tanh method.

The modified extended tanh method is developed by Malfliet in [10, 11], and used in [12-14] among many others. Since all derivatives of a tanh can be represented by tanh itself, consider the general NLPDE say in two variables

$$
H\left(u, u_{t}, u_{x}, u_{x} x, u_{t} t, u_{x} t, \ldots\right)=0 .
$$

Now we consider its travelling $u(x, t)=u(\xi)$, where $\xi=x-c t$ or $\xi=x+c t$ and the equation becomes an ordinary differential equation. We apply the following series expansion:

$$
u(\xi)=\sum_{i=0}^{N} a_{i} \phi^{i}+\sum_{i=1}^{N} b_{i} \phi^{-i}, \quad \phi^{\prime}=b+\phi^{2}
$$

where $b$ is a parameter to be determined, $\phi=\phi(\xi)$ and $\phi^{\prime}=\frac{d \phi}{d \xi}$.

To determine the parameter $\mathrm{N}$, we usually balance the linear terms of highest-order in the resulting equation with the highest-order nonlinear terms. Then we can get all coefficients of different powers of $\phi$ and determine $a_{i}, b_{i}, b, c$ by make them equals to zeros. 
The Riccati equation has the following general solutions:

(a) If $b<0$, then

$$
\phi=-\sqrt{-b} \tanh (\sqrt{-b} \xi)
$$

(b) If $b>0$, then

$$
\phi=\sqrt{b} \tan (\sqrt{b} \xi)
$$

(c) If $b=0$, then

$$
\phi=-\frac{1}{\xi}
$$

\section{Exact Travelling Wave Solutions of (1)}

We consider the travelling wave solution $u(x, y, t)=u(\xi), \xi=x+k y-c t$ for (1), and also

$$
u(\xi)=P(\xi)+i Q(\xi)
$$

According to (1) and (2), we can get

$$
i\left(P_{t}+i Q_{t}\right)+\alpha\left(P_{x x}+i Q_{x x}\right)+\beta\left(P_{y y}+i Q_{y y}\right)+r\left(P^{2}+Q^{2}\right)(P+i Q)=0 .
$$

From (3) we can obtain two equations

$$
\begin{gathered}
-Q_{t}+\alpha P_{x x}+\beta P_{y y}+r P^{3}+r Q^{2} P=0, \\
P_{t}+\alpha Q_{x x}+\beta Q_{y y}+r P^{2} Q+r Q^{3}=0 .
\end{gathered}
$$

For $u(x, y, t)=u(\xi)$ and $\xi=x+k y-c t$, we can transmute (4) into ordinary differential equations

$$
\begin{gathered}
c Q^{\prime}+\left(\alpha+\beta k^{2}\right) P^{\prime \prime}+r P^{3}+r Q^{2} P=0, \\
-c P^{\prime}+\left(\alpha+\beta k^{2}\right) Q^{\prime \prime}+r P^{2} Q+r Q^{3}=0 .
\end{gathered}
$$

The solution can be expressed as the following form 


$$
\begin{aligned}
& P(\xi)=\sum_{i=0}^{N} a_{i} \phi^{i}+\sum_{i=1}^{N} b_{i} \phi^{-i}, \\
& Q(\xi)=\sum_{i=0}^{N 1} c_{i} \phi^{i}+\sum_{i=1}^{N 1} d_{i} \phi^{-i} .
\end{aligned}
$$

Balancing the linear term of highest order with the nonlinear term in both equations, we find

$$
\begin{aligned}
& N-2+4=2 N+N 1=3 N, \\
& N 1-2+4=2 N 1+N=3 N .
\end{aligned}
$$

Thus, $N=N 1=1$, and

$$
\begin{aligned}
& P(\xi)=a_{0}+a_{1} \phi+b_{1} \phi^{-1}, \\
& Q(\xi)=c_{0}+c_{1} \phi+d_{1} \phi^{-1} .
\end{aligned}
$$

With $\phi^{\prime}=b+\phi^{2}$, we get

$$
\begin{gathered}
P^{\prime}(\xi)=\left(a_{1} b-b_{1}\right)+a_{1} \phi^{2}-b_{1} b \phi^{-2}, \\
P^{\prime \prime}(\xi)=2 a_{1} \phi^{3}+2 b a_{1} \phi+2 b^{2} b_{1} \phi^{-3}+2 b b_{1} \phi^{-1}, \\
Q^{\prime}(\xi)=\left(c_{1} b-d_{1}\right)+c_{1} \phi^{2}-d_{1} b \phi^{-2}, \\
Q^{\prime \prime}(\xi)=2 c_{1} \phi^{3}+2 b c_{1} \phi+2 b^{2} d_{1} \phi^{-3}+2 b d_{1} \phi^{-1} .
\end{gathered}
$$

Substituting (7) and (8) into two ordinary differential equations (5), and collecting the coefficients of $\phi$ gets two system of algebraic equations for $a_{0}, a_{1}, b_{1}, c_{0}, c_{1}, d_{1}$, $b, c, k$,

$$
\begin{aligned}
& \phi^{0}:-c\left(a_{1} b-b_{1}\right)+r\left(a_{0}^{2}+2 a_{1} b_{1}\right) c_{0}+2 r a_{0} b_{1} c_{1}+2 r a_{0} a_{1} d_{1} \\
&+r c_{0}\left(c_{0}^{2}+2 c_{1} d_{1}\right)+4 r c_{0} c_{1} d_{1}=0, \\
& \phi: 2\left(\alpha+\beta k^{2}\right) c_{1} b+r c_{1}\left(a_{0}^{2}+2 a_{1} b_{1}\right)+2 r c_{0} a_{0} a_{1}+r a_{1}^{2} d_{1} \\
&+2 r c_{0}^{2} c_{1}+r c_{1}\left(c_{0}^{2}+2 c_{1} d_{1}\right)+r d_{1} c_{1}^{2}=0,
\end{aligned}
$$




$$
\begin{aligned}
& \phi^{2}:-c a_{1}+r c_{0} a_{1}^{2}+2 r a_{0} a_{1} c_{1}+3 r c_{0} c_{1}^{2}=0, \\
& \phi^{3}: 2 c_{1}\left(\alpha+\beta k^{2}\right)+r c_{1} a_{1}^{2}+r c_{1}^{3}=0 \\
& \phi^{-1}: 2 d_{1} b\left(\alpha+\beta k^{2}\right)+2 r a_{0} c_{0} b_{1}+r c_{1} b_{1}^{2}+r d_{1}\left(a_{0}^{2}+2 a_{1} b_{1}\right) \\
& \quad+2 r c_{0}^{2} d_{1}+r c_{1} d_{1}^{2}+r d_{1}\left(c_{0}^{2}+2 c_{1} d_{1}\right)=0, \\
& \phi^{-2}: c b_{1} b+r c_{0} b_{1}^{2}+2 r a_{0} b_{1} d_{1}+3 r c_{0} d_{1}^{2}=0 \\
& \phi^{-3}: 2 d_{1} b^{2}\left(\alpha+\beta k^{2}\right)+r d_{1} b_{1}^{2}+r d_{1}^{3}=0
\end{aligned}
$$

and

$$
\begin{aligned}
\phi^{0}: c & \left(c_{1} b-d_{1}\right)+r a_{0}\left(a_{0}^{2}+2 a_{1} b_{1}\right)+4 r a_{0} a_{1} b_{1}+r a_{0}\left(c_{0}^{2}+2 c_{1} d_{1}\right) \\
& +2 r a_{1} c_{0} d_{1}+2 r c_{0} c_{1} b_{1}=0
\end{aligned}
$$$$
\phi: 2 a_{1} b\left(\alpha+\beta k^{2}\right)+2 r a_{0}^{2} a_{1}+r a_{1}\left(a_{0}^{2}+2 a_{1} b_{1}\right)+r a_{1}^{2} b_{1}+2 r a_{0} c_{0} c_{1}
$$$$
+r a_{1}\left(c_{0}^{2}+2 c_{1} d_{1}\right)+r b_{1} c_{1}^{2}=0,
$$$$
\phi^{2}: c c_{1}+r a_{1}^{2} a_{0}+2 r a_{0} a_{1}^{2}+2 r a_{1} c_{0} c_{1}+r a_{0} c_{1}^{2}=0,
$$$$
\phi^{3}: 2 a_{1}\left(\alpha+\beta k^{2}\right)+r a_{1}^{3}+r a_{1} c_{1}^{2}=0 \text {, }
$$$$
\phi^{-1}: 2 b_{1} b\left(\alpha+\beta k^{2}\right)+2 r a_{0}^{2} b_{1}+r a_{1} b_{1}^{2}+r b_{1}\left(a_{0}^{2}+2 a_{1} b_{1}\right)+r a_{1} d_{1}^{2}
$$$$
+2 r a_{0} c_{0} d_{1}+r b_{1}\left(c_{0}^{2}+2 c_{1} d_{1}\right)=0
$$$$
\phi^{-2}:-c b d_{1}+r a_{0} b_{1}^{2}+2 r a_{0} b_{1}^{2}+r a_{0} d_{1}^{2}+2 r c_{0} b_{1} d_{1}=0,
$$$$
\phi^{-3}: 2 b_{1} b^{2}\left(\alpha+\beta k^{2}\right)+r b_{1}^{3}+r b_{1} d_{1}^{2}=0 .
$$

With the aid of Maple, we obtain $a_{0}, a_{1}, b_{1}, c_{0}, c_{1}, d_{1}, b, c, k$ as follows:

\section{Case 1.}

$$
a_{0}=-c_{0} c_{1}\left(-\frac{2 \alpha+2 \beta k^{2}+r c_{1}^{2}}{r}\right)^{-\frac{1}{2}}, \quad a_{1}=\left(-\frac{2 \alpha+2 \beta k^{2}+r c_{1}^{2}}{r}\right)^{\frac{1}{2}},
$$




$$
\begin{gathered}
b_{1}=-\frac{c_{0}^{2}}{4}\left(-\frac{2 \alpha+2 \beta k^{2}+r c_{1}^{2}}{r}\right)^{-\frac{1}{2}}, \quad b=-\frac{1}{4} \frac{c_{0}^{2} r}{2 \alpha+2 \beta k^{2}+r c_{1}^{2}}, \\
c=-2 c_{0}\left(\alpha+\beta k^{2}\right)\left(-\frac{2 \alpha+2 \beta k^{2}+r c_{1}^{2}}{r}\right)^{-\frac{1}{2}}, \quad c_{0}=c_{0}, \quad c_{1}=c_{1}, \\
d_{1}=\frac{1}{4} \frac{c_{0}^{2} c_{1} r}{2 \alpha+2 \beta k^{2}+r c_{1}^{2}}, \quad k=k,
\end{gathered}
$$

where $r, \beta, \alpha, k, c_{0}, c_{1}$ are arbitrary constants.

\section{Case 2.}

$$
\begin{gathered}
a_{0}=-d_{1}\left(-\frac{2 \alpha+2 \beta k^{2}}{r d_{1}^{2}+r b_{1}^{2}}\right)^{\frac{1}{4}}, \quad a_{1}=0, \quad b_{1}=b_{1}, \quad b=\left(-\frac{2 \alpha+2 \beta k^{2}}{r d_{1}^{2}+r b_{1}^{2}}\right)^{-\frac{1}{4}}, \\
c=-r\left(d_{1}^{2}+b_{1}^{2}\right)\left(-\frac{2 \alpha+2 \beta k^{2}}{r d_{1}^{2}+r b_{1}^{2}}\right)^{\frac{3}{4}}, \quad c_{0}=b_{1}\left(-\frac{2 \alpha+2 \beta k^{2}}{r d_{1}^{2}+r b_{1}^{2}}\right)^{\frac{1}{4}}, \\
c_{1}=0, \quad d_{1}=d_{1}, \quad k=k,
\end{gathered}
$$

where $r, \beta, \alpha, k, b_{1}, d_{1}$ are arbitrary constants.

\section{Case 3.}

$$
\begin{gathered}
a_{0}=\left(-\frac{2 \alpha d_{1}^{2}+2 d_{1}^{2} \beta k^{2}}{r}\right)^{\frac{1}{4}}, \quad a_{1}=0, \quad b_{1}=0, \\
b=d_{1}^{2}\left(-\frac{2 \alpha d_{1}^{2}+2 d_{1}^{2} \beta k^{2}}{r}\right)^{-\frac{1}{2}}, \quad c=\frac{r}{d_{1}}\left(-\frac{2 \alpha d_{1}^{2}+2 d_{1}^{2} \beta k^{2}}{r}\right)^{\frac{3}{4}}, \\
c_{0}=0, \quad c_{1}=0, \quad d_{1}=d_{1}, \quad k=k,
\end{gathered}
$$

where $r, \beta, \alpha, k, d_{1}$ are arbitrary constants. 


\section{Case 4.}

$$
\begin{gathered}
a_{0}=a_{0}, \quad a_{1}=0, \quad b_{1}=0, \quad b=-\frac{1}{2} \frac{r a_{0}^{2}}{\alpha+\beta k^{2}}, \\
c=-r a_{0}\left(-\frac{2 \alpha+2 \beta k^{2}}{r}\right)^{\frac{1}{2}}, \quad c_{0}=0, \quad c_{1}=\left(-\frac{2 \alpha+2 \beta k^{2}}{r}\right)^{\frac{1}{2}}, \quad d_{1}=0, \quad k=k,
\end{gathered}
$$

where $r, \beta, \alpha, k, a_{0}$ are arbitrary constants.

\section{Case 5.}

$$
\begin{gathered}
a_{0}=a_{0}, \quad a_{1}=0, \quad b_{1}=0, \quad b=-\frac{1}{8} \frac{r a_{0}^{2}}{\alpha+\beta k^{2}}, \\
c=-r a_{0}\left(-\frac{2 \alpha+2 \beta k^{2}}{r}\right)^{\frac{1}{2}}, \quad c=-r a_{0}\left(-\frac{4+2 \beta k^{2}}{r}\right)^{\frac{1}{2}}, \quad c_{0}=0, \\
c_{1}=\left(-\frac{2 \alpha+2 \beta k^{2}}{r}\right)^{\frac{1}{2}}, \quad d_{1}=-\frac{a_{0}^{2}}{4}\left(-\frac{2 \alpha+2 \beta k^{2}}{r}\right)^{-\frac{1}{2}}, \quad k=k,
\end{gathered}
$$

where $r, \beta, \alpha, k, a_{0}$ are arbitrary constants.

\section{Case 6.}

$$
\begin{aligned}
& a_{0}=0, \quad a_{1}=\left(-\frac{2 \alpha+2 \beta k^{2}}{r}\right)^{\frac{1}{2}}, \quad b_{1}=0, \quad b=-\frac{1}{2} \frac{r c_{0}^{2}}{\alpha+\beta k^{2}}, \\
& c=r c_{0}\left(-\frac{2 \alpha+2 \beta k^{2}}{r}\right)^{\frac{1}{2}}, \quad c_{0}=c_{0}, \quad c_{1}=0, \quad d_{1}=0, k=k,
\end{aligned}
$$

where $r, \beta, \alpha, k, c_{0}$ are arbitrary constants.

\section{Case 7.}

$$
a_{0}=-c_{0} c_{1}\left(-\frac{2 \alpha+2 \beta k^{2}+r c_{1}^{2}}{2}\right)^{-\frac{1}{2}}, \quad a_{1}=\left(-\frac{2 \alpha+2 \beta k^{2}+r c_{1}^{2}}{2}\right)^{\frac{1}{2}},
$$




$$
\begin{gathered}
b_{1}=0, \quad b=\frac{-c_{0}^{2} r}{2 \alpha+2 \beta k^{2}+r c_{1}^{2}}, \quad c=-2\left(\alpha+\beta k^{2}\right) c_{0}\left(-\frac{2 \alpha+2 \beta k^{2}+r c_{1}^{2}}{2}\right)^{-\frac{1}{2}}, \\
c_{0}=c_{0}, \quad c_{1}=c_{1}, \quad d_{1}=0, \quad k=k
\end{gathered}
$$

where $r, \beta, \alpha, k, c_{0}, c_{1}$ are arbitrary constants.

\section{Case 8.}

$$
\begin{gathered}
a_{0}=0, \quad a_{1}=\left(-\frac{2 \alpha+2 \beta k^{2}}{r}\right)^{\frac{1}{2}}, \quad b_{1}=\frac{1}{8} \frac{r c_{0}^{2}}{\alpha+\beta k^{2}}\left(-\frac{2 \alpha+2 \beta k^{2}}{r}\right)^{\frac{1}{2}}, \\
b=-\frac{1}{8} \frac{r c_{0}^{2}}{\alpha+\beta k^{2}}, \quad c=r c_{0}\left(-\frac{2 \alpha+2 \beta k^{2}}{r}\right)^{\frac{1}{2}}, \\
c_{0}=c_{0}, \quad c_{1}=0, \quad d_{1}=0, \quad k=0,
\end{gathered}
$$

where $r, \beta, \alpha, k, c_{0}$ are arbitrary constants.

\section{Case 9.}

$$
\begin{aligned}
& a_{0}=0, \quad a_{1}=0, \quad b_{1}=c_{0}^{2} \sqrt{-\frac{r}{2 \alpha+2 \beta k^{2}}}, \quad b=-\frac{1}{2} \frac{c_{0}^{2} r}{\alpha+\beta k^{2}}, \\
& c=-\frac{c_{0} r}{\sqrt{-\frac{r}{2 \alpha+2 \beta k^{2}}}}, \quad k=k, \quad c_{0}=c_{0}, \quad c_{1}=0, \quad d_{1}=0,
\end{aligned}
$$

where $r, \beta, \alpha, k, c_{0}$ are arbitrary constants.

If $b>0$, we get

$$
u=a_{0}+a_{1} v(x, y, t)+b_{1} v(x, y, t)^{-1}+i\left(c_{0}+c_{1} v(x, y, t)+d_{1} v(x, y, t)^{-1}\right),
$$

where $v(x, y, t)=\sqrt{b} \tan (\sqrt{b}(x+k y-c t))$. 
If $b=0$, we get

$$
u=a_{0}+a_{1} v(x, y, t)+b_{1} v(x, y, t)^{-1}+i\left(c_{0}+c_{1} v(x, y, t)+d_{1} v(x, y, t)^{-1}\right),
$$

where $v(x, y, t)=\frac{1}{x+k y-c t}$.

If $b<0$, we get

$$
u=a_{0}+a_{1} v(x, y, t)+b_{1} v(x, y, t)^{-1}+i\left(c_{0}+c_{1} v(x, y, t)+d_{1} v(x, y, t)^{-1}\right),
$$

where $v(x, y, t)=-\sqrt{-b} \tanh (\sqrt{-b}(x+k y-c t))$.

Substituting all those situations into (9) respectively, we can get all solutions of the derivative nonlinear Schrödinger equation.

\section{Image Simulation}

In order to grasp these exact travelling solutions, we choose several exact solutions and use the Matlab software to simulate images. In the process of image simulation, the figures and value of parameters we selected show as follows:

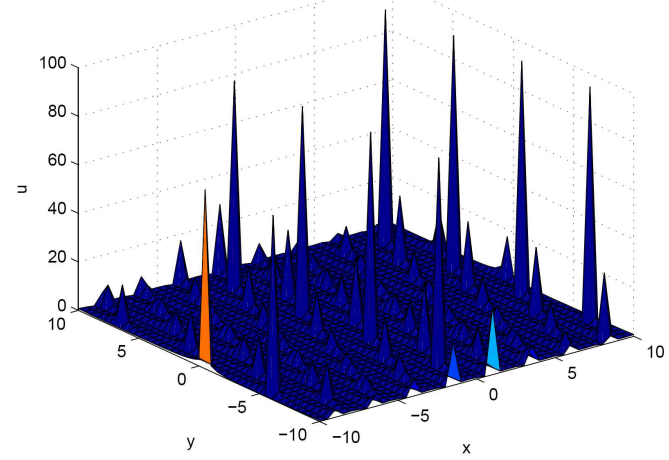

Figure 1. 3-dimensional wave of Case 1.

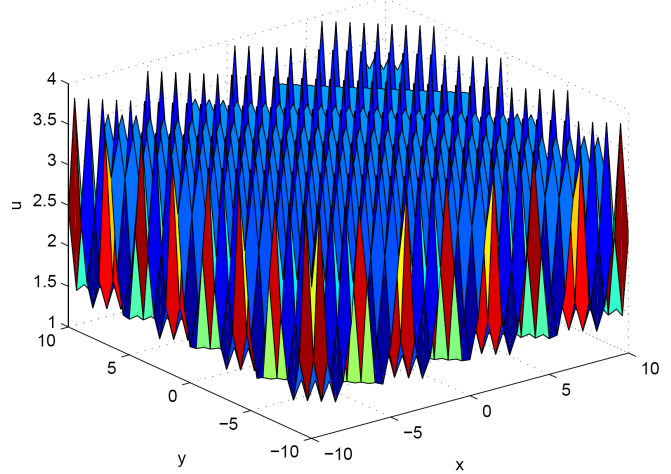

Figure 2. 3-dimensional wave of Case 2.

In Figure 1, we take $r=-1, \quad \beta=11, k=0.1, \quad c_{0}=-0.25, \quad c_{1}=0.1, \quad \alpha=-0.2$, $t=0.1$. In Figure 2, we take $r=-6, \beta=-3, k=1, d_{1}=1, \alpha=1, b_{1}=-1, t=0.1$. 


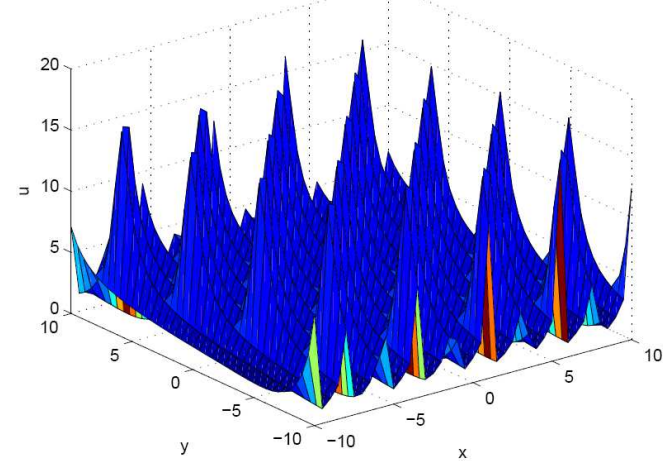

Figure 3. 3-dimensional wave of Case 3.

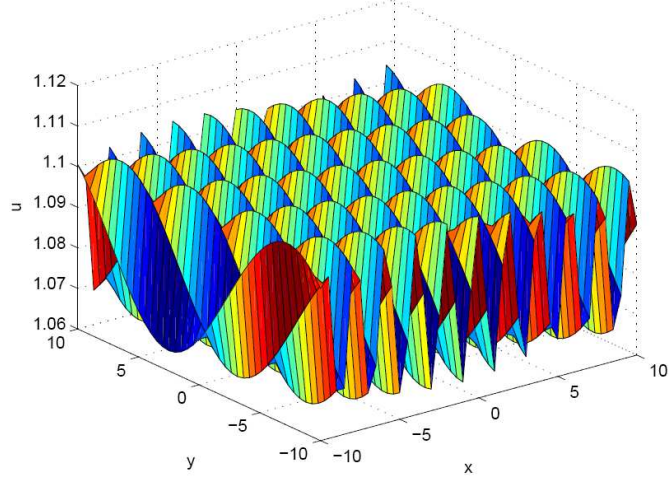

Figure 4. 3-dimensional wave of Case 3.

In Figure 3, we take $r=0.1, \beta=0.3, k=0.1, d_{1}=-2, \alpha=0.2, t=0.1$. In Figure 4, we take $r=-6, \beta=2, k=0.1, c_{0}=6, \alpha=-4, d_{1}=2, t=0.1$.

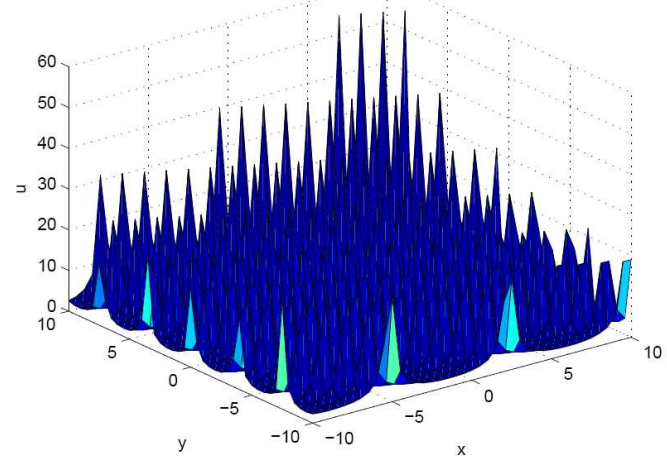

Figure 5. 3-dimensional wave of Case 4.

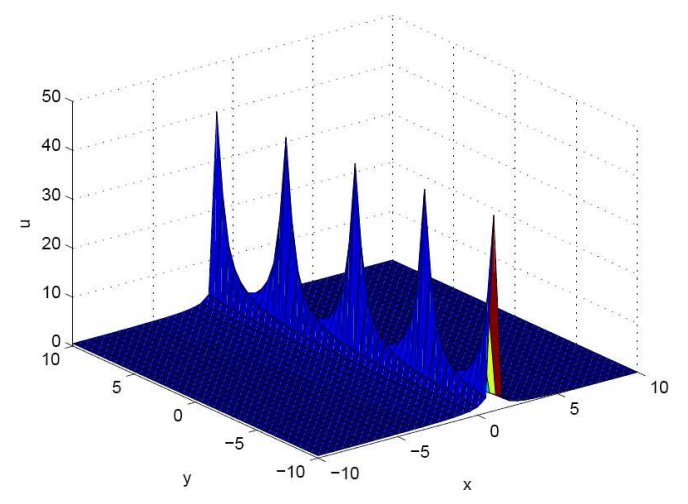

Figure 6. 3-dimensional wave of Case 5.

In Figure 5, we take $r=-1, \beta=3, k=3, a_{0}=2, \alpha=-0.7, t=0.1$. In Figure 6, we take $r=-1, \beta=-0.3, k=0.1, a_{0}=-0.25, \alpha=-1, t=0.1$. 


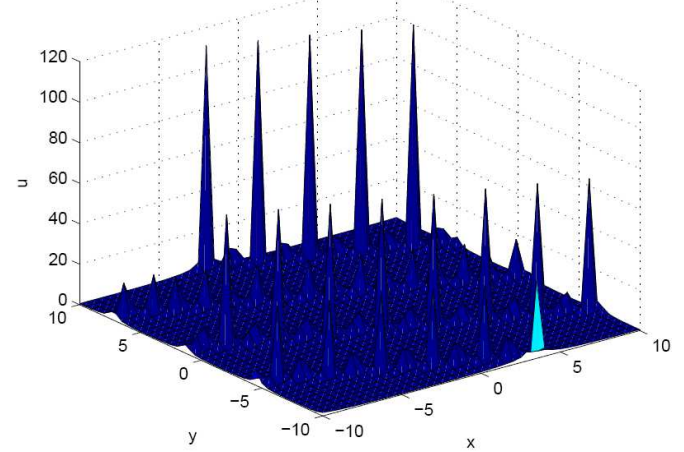

Figure 7. 3-dimensional wave of Case 6.

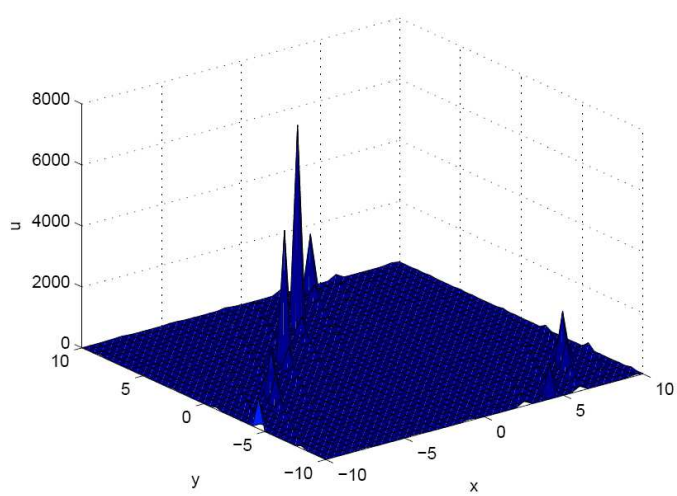

Figure 8. 3-dimensional wave of Case 7.

In Figure 7, we take $r=-6, \beta=3, k=2.5, c_{0}=-0.5, \alpha=-2, t=0.1$. In Figure 8, we take $r=-1, \beta=3, k=2.9, c_{0}=6, \alpha=-2, c_{1}=2, t=0.1$.

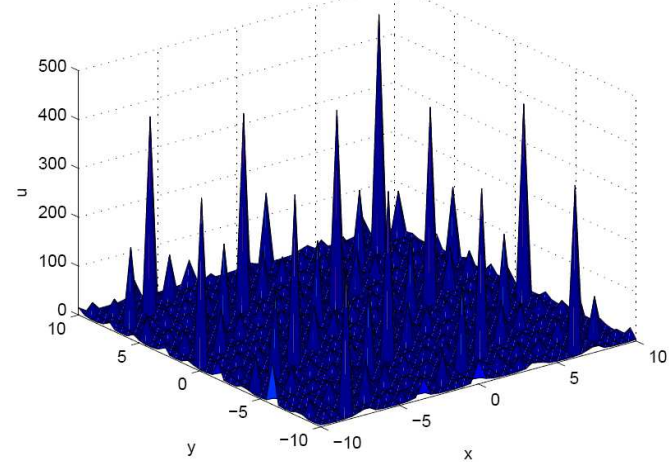

Figure 9. 3-dimensional wave of Case 8.

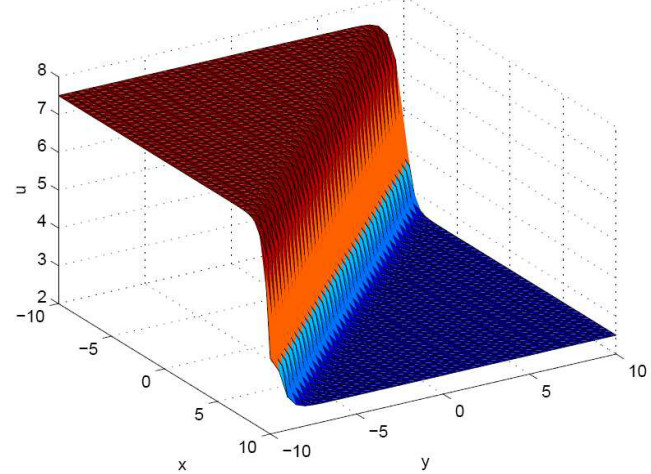

Figure 10. 3-dimensional wave of Case 9.

In Figure 9, we take $r=-2, \beta=2, k=1.6, c_{0}=2.5, \alpha=3.2, t=0.1$. In Figure 10 , we take $r=-0.1, \beta=1, k=1, c_{0}=2.5, \alpha=-1, c_{1}=1, t=0.1$. 


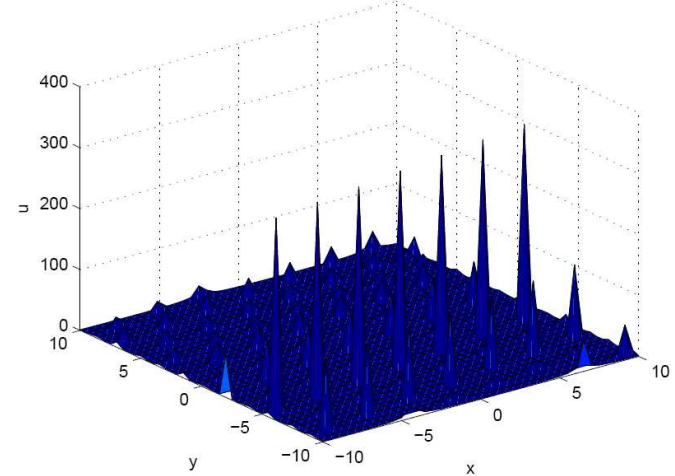

Figure 11. 3-dimensional wave of Case 9 .

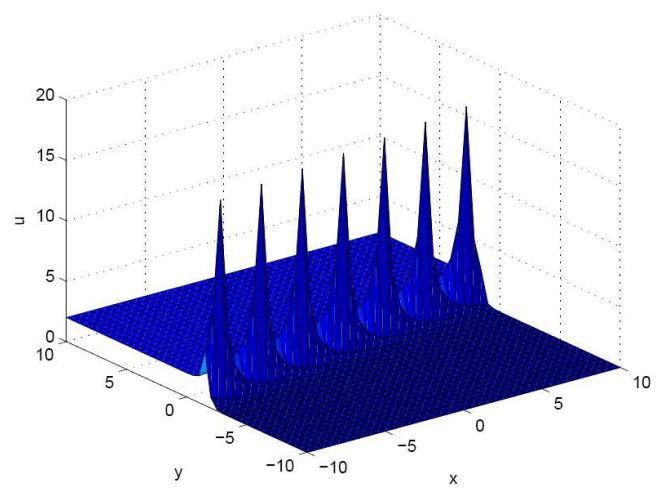

Figure 12. 3-dimensional wave of Case 9.

In Figure 11, we take $r=-6, \beta=1, k=-6, c_{0}=1, \alpha=-1, t=0.1$. In Figure 12, we take $r=6, \beta=1, k=-6, c_{0}=1, \alpha=-1, t=0.01$.

\section{Conclusion}

In this paper we studied the nonlinear Schrödinger equation by finding its exact travelling wave solutions through the modified extended tanh methods. With the aid of waveform graphs of the solutions, we can obtain the related properties of the equation. However, we can also use the method of dynamical systems to obtain the bounded solutions.

\section{References}

[1] P. Gray and S. Scott, Chemical Oscillations and Instabilities, Oxford: Clarendon Press, 1990.

[2] J. Murray, Mathematical Biology, Berlin: Springer, 1989.

[3] Ding-jiang Huang, De-sheng Li and Hong-qing Zhang, Explicit and exact travelling wave solutions for the generalized derivative Schrödinger equation, Chaos Solitons Fractals 31 (2007), 586-593.

[4] V.O. Vakhnenko, E.J. Parkes, A.J. Morrison, A Bäcklund transformation and the inverse scattering transform method for the generalized Vakhnenko equation, Chaos Solitons Fractals 17 (2003), 683-692.

[5] E. Yomba, The extended Fan's sub-equation method and its application to KdV-MKdV, BKK and variant Boussinesq equations, Phys. Lett. A 336 (2005), 463-476. 
[6] V.B. Matveev and M.A. Salle, Darboux Transformation and Solitons, Berlin: Springer, 2001.

[7] S.A. Khuri, A complex tanh-function method applied to nonlinear equations of Schrödinger type, Chaos Solitons Fractals 20 (2004), 1037-1040.

[8] L. Kavitha, N. Akila, A. Prabhu, O. Kuzmanovska-Barandovska and D. Gopi, Exact solitary solutions of an inhomogeneous modified nonlinear Schrödinger equation with competing nonlinearities, Math. Comput. Modelling 53 (2011), 1095-1110.

[9] Engui Fan and Y.C. Hon, Applications of extended tanh method to "special" types of nonlinear equations, Appl. Math. Comput. 141 (2003), 351-358.

[10] W. Malfliet, Solitary wave solutions of nonlinear wave equations, Amer. J. Phys. 60 (1992), 650-654.

[11] W. Malfliet, The tanh method: a tool for solving certain classes of nonlinear evolution and wave equations, J. Comput. Appl. Math. 164/165 (2004), 529-541.

[12] Abdul-Majid Wazwaz, The Camassa-Holm-KP equations with compact and noncompact travelling wave solutions, Appl. Math. Comput. 170 (2005), 347-360.

[13] A.-M. Wazwaz, The tanh and the sine-cosine methods for the complex modified KdV and the generalized KdV equations, Comput. Math. Appl. 49 (2005), 1101-1112.

[14] Abdul-Majid Wazwaz, The extended tanh method for abundant solitary wave solutions of nonlinear wave equations, Appl. Math. Comput. 187 (2007), 1131-1142.

[15] Juan Wang, Longwei Chen and Changfu Liu, Bifurcations and travelling wave solutions of a (2+1)-dimensional nonlinear Schrödinger equation, Appl. Math. Comput. 249 (2014), $76-80$. 